\title{
On the stability of pairwise triangularisable and related switching systems
}

\author{
Robert Shorten \\ Department of Computer Science \\ National University of Ireland \\ Maynooth, Co. Kildare \\ Ireland
}

\author{
Fiacre Ó Cairbre \\ Department of Mathematics \\ National University of Ireland \\ Maynooth, Co. Kildare \\ Ireland
}

\begin{abstract}
It was recently conjectured in [1] that a sufficient condition for the asymptotic stability of the switching system $\dot{x}=A(t) x, A(t) \in \mathcal{A} \triangleq\left\{A_{1}, \ldots, A_{m}\right\}$, $A_{i} \in \mathbb{R}^{n \times n}$, is that the component systems $\Sigma_{A_{i}}$ : $\dot{x}=A_{i} x, i \in\{1, \ldots, m\}$ are asymptotically stable, and a set of non-singular matrices $T_{i j}$ exist such that the matrices $\left\{T_{i j} A_{i} T_{i j}^{-1}, T_{i j} A_{j} T_{i j}^{-1}\right\}, i, j \in$ $\{1, \ldots, m\}$, are upper triangular (pairwise triangular). In this paper we show that while the general condition of pairwise triangularisability is not sufficient to guarantee asymptotic stability, the ideas developed in [1] can be extended to prove asymptotic stability of the origin for a related class of switching system.
\end{abstract}

\section{Introductory remarks}

We consider switching systems of the following form,

$$
\dot{x}=A(t) x, A(t) \in \mathcal{A} \triangleq\left\{A_{1}, \ldots, A_{m}\right\},
$$

where the $A_{i}, i \in\{1,2, \ldots m\}$, are constant matrices in $\mathbb{R}^{n \times n}$. The matrices $A_{i}, i \in\{1,2, \ldots m\}$, are assumed to be Hurwitz. A sufficient condition for the exponential stability of (1) is that the matrices in $\mathcal{A}$ are simultaneously triangularisable. Unfortunately, from a practical viewpoint, the requirement of simultaneous triangularisability imposes unrealistic conditions on the matrices in the set $\mathcal{A}$. In [1] we considered classes of systems where a set of non-singular matrices $T_{i j}$ exist such that the matrices $\left\{T_{i j} A_{i} T_{i j}^{-1}, T_{i j} A_{j} T_{i j}^{-1}\right\}, i, j \in\{1, \ldots, m\}$, are upper triangular (pairwise triangularisable switching systems). Here, we show that while the condition of pairwise triangularisability is not sufficient to guarantee asymptotic stability, the techniques introduced for their study in $[1,2]$, can be used to prove the global attractivity of related systems.

\section{Main result}

The condition of pairwise triangularisability was conjectured to be sufficient for asymptotic stability of (1) in [1]. However, this conjecture was demonstrated to be not true by means of a simple example in [4]. In [4] an example of an unstable systems was presented that satisfies the condition of pairwise triangularisability.

Here we consider a natural extension of the system class studied in [1]; namely, switching systems that are constructed with the property that any pair of system matrices are Hurwitz and diagonalisable, and in addition share at least $n-1$ common conjugate pairs of complex eigenvectors.

Preamble for Theorem 4.1: Let $\mathcal{V}=$ $\left\{v_{1}, v_{1}^{*}, v_{2}, v_{2}^{*}, \ldots, v_{n+1}, v_{n+1}^{*}\right\}$ be a set of complex (non-real) vectors in $\mathbb{C}^{2 n}$. Here $*$ denotes the complex conjugate. Suppose any $2 n$ vectors of the form $v_{1}, v_{1}^{*}, \ldots, v_{i-1}, v_{i-1}^{*}, v_{i+1}^{*}$, $v_{i+1}^{*}, \ldots, v_{n+1}, v_{n+1}^{*}$, where both $v_{i}$ and $v_{i}^{*}$ are omitted, are linearly independent in $\mathbb{C}^{2 n}$ (over $\mathbb{C})$, for any $i \in\{1,2, \ldots, n+1\}$. For each $i \in\{1,2, \ldots, n+1\}$, construct a $2 n \times 2 n$ matrix as follows: $M_{1}=\left[v_{1}, v_{1}^{*}, v_{2}, v_{2}^{*}, \ldots, v_{n}, v_{n}^{*}\right]$ and for $2 \leq i \leq n+1$ we define $M_{i}=\left[v_{1}, v_{1}^{*}, \ldots ; v_{n+1}\right.$, $\left.v_{n+1}^{*}, v_{i}, v_{i}^{*}, \ldots, v_{n}, v_{n}^{*}\right]$, i.e. $M_{i}$ is obtained by replacing the $v_{i-1}$ and $v_{i-1}^{*}$ columns by $v_{n+1}$ and $v_{n+1}^{*}$ respectively. Thus, the columns of each $M_{j}$ are linearly independent and so $M_{j}$ is invertible. Suppose we also have $p$ different $2 n \times 2 n$ diagonal matrices $D_{1}, D_{2}, \ldots, D_{p}$ with all diagonal entries negative. In each of these diagonal matrices we assume that the $(k, k)$ entry equals the $(k-1, k-1)$ entry whenever $k$ is even.

Suppose $A_{i, h}=M_{i} D_{h} M_{i}^{-1}$ has real entries for $1 \leq i \leq n+1$ and $1 \leq h \leq p$. In other words, $A_{i, h}$ is a real matrix with complex (non-real) eigenvectors (occurring in conjugate pairs) lying in $\mathcal{V}$ and 
negative eigenvalues. This gives us $m$ different diagonalisable matrices $A_{i, h}$, where $m=p(n+1)$. Let $\mathcal{A}$ be a subset of $\left\{A_{i, h}: 1 \leq i \leq n+1,1 \leq h \leq p\right\}$. Thus, $\mathcal{A}$ is a set of at most $m$ different diagonalisable matrices such that any two matrices in $\mathcal{A}$ have at least $n-1$ linearly independent common conjugate pairs of complex eigenvectors.

Statement of Theorem 4.1: If we consider the switching system (1) with the set $\mathcal{A}$ defined as in the above preamble, then the origin is globally attractive. Thus, asymptotic stability follows from [3].

Proof: For details of the proof the reader is referred to [4]. Here we present an outline of the main ideas only.

Step 1: We replace each $2 n \times 2 n$ matrix $M_{i}$ by a $(2 n+2) \times(2 n+2)$ matrix $\bar{M}_{i}$. We then replace each $2 n \times 2 n$ matrix $A_{i, h}$ in $\mathcal{A}$ by a $(2 n+2) \times$

$(2 n+2)$ matrix $\bar{A}_{i, h}$. The matrices $\bar{A}_{i, h} \in \overline{\mathcal{A}} \triangleq$ $\left\{\bar{A}_{i, h}: A_{i, h} \in \mathcal{A}\right\}$ are chosen such that there is at least one conjugate pair of common eigenvectors, $\left(a_{1}, a_{2}, 0, \ldots, 0\right),\left(a_{1}^{*}, a_{2}^{*}, 0, \ldots, 0\right)$, for all the matrices in $\overline{\mathcal{A}}$, and also such that the properties of the solutions of the dynamic system,

$$
\dot{\bar{x}}=\bar{A}(t) \bar{x}, \bar{A}(t) \in \overline{\mathcal{A}},
$$

will ultimately imply the global attractivity of the origin of the system,

$$
\begin{aligned}
& \dot{x}=A(t) x, A(t) \in \mathcal{A}, \\
& \text { where } x=\left(x_{1}, x_{2}, \ldots, x_{2 n}\right) \text { and } \bar{x}= \\
& \left(x_{2 n+2}, x_{2 n+1}, x_{1}, x_{2}, \ldots, x_{2 n}\right) .
\end{aligned}
$$

Step 2 : For a given $j \in\{1,2, \ldots, n+1\}$ we consider the $2 n+2$ linearly independent columns of $\bar{M}_{j}$. These form a $2 n+2$ dimensional coordinate system which includes $\left(a_{1}, a_{2}, 0, \ldots, 0\right)$ as one of the axes. We consider the projection of the state $\bar{x}(t)$ onto $\left(a_{1}, a_{2}, 0, \ldots, 0\right)$ as the dynamics of the system (2) evolve. This projection is given by the first component of the vector,

$$
g_{j}(t)=\bar{M}_{j}^{-1} \bar{x}(t),
$$

and is denoted by $\left[g_{j}\right]_{1}(t)$.

Step 3: We then show that,

$$
\begin{aligned}
& \lim _{t \rightarrow \infty}\left|\operatorname{Re}\left[g_{i}\right]_{1}(t)-\operatorname{Re}\left[g_{j}\right]_{1}(t)\right|=0 \\
& =\lim _{t \rightarrow \infty}\left|\operatorname{Im}\left[g_{i}\right]_{1}(t)-\operatorname{Im}\left[g_{j}\right]_{1}(t)\right|,
\end{aligned}
$$

for all $i, j \in\{1,2, \ldots, n+1\}$. Note that $R e$ denotes the real part of a complex number and $I m$ denotes the imaginary part. From the above fact we can deduce that $\lim _{t \rightarrow \infty}\left(x_{1}, x_{2}, \ldots, x_{2 n}\right)=0$. This is sufficient to prove the global attractivity of the origin of the system,

$$
\dot{x}=A(t) x, A(t) \in \mathcal{A} .
$$

\section{Concluding remarks}

In this paper we have considered a class of systems related to the system class studied in [1]. The tools developed for the study of pairwise triangularisable swtching systems are useful for the study of this class of system, and are used to prove global attractivity of the origin. The technique that is utilised in this paper involves proving global attractivity by embedding the original ( $n$-dimensional) state space in a higher dimensional state space and does not employ arguments from quadratic Lyapunov theory. It is likely that this methodology is applicable to other classes of switching systems.

\section{Acknowledgements}

This work was partially supported by the European Union funded research training network Multi-Agent Contral, HPRN-CT-1999-00107 and by the Enterprise Ireland grant $\mathrm{SC} / 2000 / 084 / \mathrm{Y}$. This work is the sole responsibility of the authors and does not refiect the European Union's opinion. The EU is not responsible for any use of data appearing in this publication.

\section{References}

[1] R. Shorten and F Ó Cairbre. On the global attractivity of a class of switching systems. In Proceedings of the American Control Conference, 2000.

[2] R. Shorten and F. Ó Cairbre. A proof of global attractivity for a class of switching systems using a non-Lyapunov Approach. Accepted for publication by Institute of Mathematics and its Applications : Journal of Mathematical Control and Information.

[3] D. Angeli. A note on the stability of arbitrarily switched homogeneous systems. Submitted to systems and control letters, 1999.

[4] F. O Cairbre and R. Shorten. On the stability of pairwise triangularisable and related switching systems. Technical report, National University of Ireland, Maynooth, NUIM/SS/RS/01/05. 